\title{
Weak Ergodicity Breaking of Receptor Motion in Living Cells Stemming from Random Diffusivity
}

\author{
Carlo Manzo, ${ }^{1, *}$ Juan A. Torreno-Pina, ${ }^{1}$ Pietro Massignan,,${ }^{1}$ Gerald J. Lapeyre, Jr., ${ }^{1}$ \\ Maciej Lewenstein, ${ }^{1,2}$ and Maria F. Garcia Parajo ${ }^{1,2, \dagger}$ \\ ${ }^{1}$ ICFO-Institut de Ciències Fotòniques, Mediterranean Technology Park, \\ 08860 Castelldefels (Barcelona), Spain \\ ${ }^{2}$ ICREA-Institució Catalana de Recerca i Estudis Avançats, 08010 Barcelona, Spain
}

(Received 27 September 2014; published 25 February 2015)

\begin{abstract}
Molecular transport in living systems regulates numerous processes underlying biological function. Although many cellular components exhibit anomalous diffusion, only recently has the subdiffusive motion been associated with nonergodic behavior. These findings have stimulated new questions for their implications in statistical mechanics and cell biology. Is nonergodicity a common strategy shared by living systems? Which physical mechanisms generate it? What are its implications for biological function? Here, we use single-particle tracking to demonstrate that the motion of dendritic cell-specific intercellular adhesion molecule 3-grabbing nonintegrin (DC-SIGN), a receptor with unique pathogen-recognition capabilities, reveals nonergodic subdiffusion on living-cell membranes In contrast to previous studies, this behavior is incompatible with transient immobilization, and, therefore, it cannot be interpreted according to continuous-time random-walk theory. We show that the receptor undergoes changes of diffusivity, consistent with the current view of the cell membrane as a highly dynamic and diverse environment. Simulations based on a model of an ordinary random walk in complex media quantitatively reproduce all our observations, pointing toward diffusion heterogeneity as the cause of DC-SIGN behavior. By studying different receptor mutants, we further correlate receptor motion to its molecular structure, thus establishing a strong link between nonergodicity and biological function. These results underscore the role of disorder in cell membranes and its connection with function regulation. Because of its generality, our approach offers a framework to interpret anomalous transport in other complex media where dynamic heterogeneity might play a major role, such as those found, e.g., in soft condensed matter, geology, and ecology.
\end{abstract}

DOI: 10.1103/PhysRevX.5.011021

\section{INTRODUCTION}

Cell function relies heavily on the occurrence of biochemical interactions between specific molecules. Encounters between interacting species are mediated by molecular transport within the cellular environment. A fundamental mode of transport for molecules in living cells is represented by diffusion, a motion characterized by random displacements. The quantitative study of diffusion is thus essential for understanding molecular mechanisms underlying cellular function, including target search [1], kinetics of transport-limited reactions [2,3], trafficking, and signaling [4]. These processes take place in complex environments, crowded and compartmentalized by macromolecules and biopolymers. A prototypical example is the

\footnotetext{
*carlo.manzo@icfo.es

†maria.garcia-parajo@icfo.es
}

Published by the American Physical Society under the terms of the Creative Commons Attribution 3.0 License. Further distribution of this work must maintain attribution to the author(s) and the published article's title, journal citation, and DOI.
Subject Areas: Biological Physics, Statistical Physics

plasma membrane, where the interplay of lipids and proteins with cytosolic (e.g., the actin cytoskeleton) and extracellular (e.g., glycans) components generates a highly dynamic and heterogeneous organization [5].

The diffusion of a single molecule $j$, whose position $x_{j}$ is sampled at $N$ discrete times $t_{i}=i \Delta t$, is often characterized by the time-averaged mean-square displacement (tMSD):

$$
\operatorname{tMSD}\left(t_{\text {lag }}=m \Delta t\right)=\frac{1}{N-m} \sum_{i=1}^{N-m}\left[x_{j}\left(t_{i}+m \Delta t\right)-x_{j}\left(t_{i}\right)\right]^{2},
$$

which for a Brownian particle scales linearly in the time lag $t_{\text {lag }}$. Application of fluorescence-based techniques to living cells has evidenced striking deviations from Brownian behavior in the nucleus [6], cytoplasm [7-10], and plasma membrane [11,12]. Indeed, numerous cellular components show anomalous subdiffusion [13], characterized by a power-law dependence of the mean-square displacement (MSD) $\sim t^{\beta}$, with $\beta<1$ [14-16]. Owing to the implications of molecular transport for cellular function and the widespread evidence of subdiffusion in biology, major 
theoretical efforts have been devoted to understand its physical origin. Subdiffusion is generally understood to be the consequence of molecular crowding [17], and several models have been developed to capture its main features. In general, subdiffusion can be obtained by models of energetic and/or geometric disorder, such as (i) the continuous-time random walk (CTRW), i.e., a walk with waiting times between steps drawn from a power-law distribution [18]; (ii) fractional Brownian motion, i.e., a process with correlated increments [19]; (iii) obstructed diffusion, i.e., a walk on a percolation cluster or a fractal [15]; and (iv) diffusion in a spatially and/or temporally heterogeneous medium [20-22]. Some of these models have been associated with relevant biophysical mechanisms such as trapping [23], the viscoelastic properties of the environment [24], or the presence of barriers and obstacles to diffusion [25].

Advances in single-particle-tracking (SPT) techniques have allowed the recording of long single-molecule trajectories and have revealed very complex diffusion patterns in living-cell systems [5,11]. Recently, it has been shown that some cellular components show subdiffusion associated with weak ergodicity breaking (WEB) $[9,10,12]$, with the most obvious signatures being the nonequivalence of the tMSD and the ensemble-averaged MSD (eMSD). The experimentally determined ensemble-averaged MSD over a time interval $m \Delta t$ is defined by

$$
\operatorname{eMSD}\left(t_{\mathrm{lag}}=m \Delta t\right)=\frac{1}{J} \sum_{j=1}^{J}\left[x_{j}\left(t_{i}+m \Delta t\right)-x_{j}\left(t_{i}\right)\right]^{2},
$$

where $J$ is the number of observed single-particle trajectories and $t_{i}$ is the starting time relative to the first point in the trajectory.

Moreover, ergodicity breaking has been further confirmed by the presence of aging [26,27], i.e., the dependence of statistical quantities on the observation time. Based on these findings, several stochastic models presenting nonstationary (and thus nonergodic) subdiffusion have been proposed [20,28-31]. Among these models, CTRW has been used to model nonergodic subdiffusion in living cells $[9,10,12]$ and has begun to provide theoretical insight into the physical origin of WEB in biological systems [28], associating the nonergodic behavior with the occurrence of particle immobilization with a heavy-tailed distribution of trapping times.

At the same time, these intriguing findings have generated new questions: Is nonergodic subdiffusion a strategy shared by other biological systems? Can biophysical mechanisms other than trapping lead to similar behaviors? What is its functional relevance? Elucidating these issues is crucial to unravel the role of nonergodic subdiffusion in cellular function. The main aim of the present work is to explore other forms of transport in biological systems to provide answers to these questions.
Here, we use SPT to study the diffusion of a prototypical transmembrane protein, the pathogen-recognition receptor dendritic cell-specific intercellular adhesion molecule 3-grabbing nonintegrin (DC-SIGN) [32], on living-cell membranes. Our experiments and data analysis show that DC-SIGN dynamics display clear signatures of WEB and aging. However, in contrast to recent studies reporting nonergodic behavior of other membrane proteins [12], we find that DC-SIGN very rarely shows trapping events so that the observed WEB cannot be described by the CTRW model. Instead, our analysis shows that DC-SIGN displays a heterogeneous dynamics presenting frequent changes of diffusivity. Our numerical simulations, based on a novel theoretical model of Brownian diffusion in complex media [21], quantitatively reproduce DC-SIGN dynamics demonstrating that nonergodic subdiffusion is a consequence of temporal and/or spatial heterogeneity. Furthermore, structurally mutated variants of DC-SIGN, with impaired function, show very different dynamical features. These results allow us to link receptor transport to molecular structure and receptor function, such as the capability to capture and uptake pathogens.

\section{WEAK ERGODICITY BREAKING AND AGING IN DC-SIGN DYNAMICS}

In this work, we perform SPT experiments [5] to follow the lateral diffusion of the pathogen-recognition receptor DC-SIGN [32] on living-cell membranes. DC-SIGN is a protein exclusively expressed on the surface of cells of the immune system, such as dendritic cells. The receptor is involved in the binding and uptake of a broad range of pathogens such as HIV-1, the Ebola virus, the hepatitis C virus, Candida albicans, and Mycobacterium tuberculosis [33]. Previous studies have shown that DC-SIGN expressed on the membrane of Chinese hamster ovary $(\mathrm{CHO})$ cells reproduces the essential features of the receptors naturally occurring on dendritic cells [34,35], thus serving as a valid model system. To characterize its dynamics, we perform video microscopy of quantum-dot-labeled DC-SIGN stably transfected in $\mathrm{CHO}$ cells in an epi-illumination configuration [Figs. 1(a) and 1(b); see Appendix A for details on cell culture and labeling procedures]. In order to follow the standard biology nomenclature and to differentiate it from its mutated forms, in this manuscript, we refer to the full receptor as the wild-type DC-SIGN (WT DC-SIGN).

We track quantum-dot positions with nanometer accuracy by means of an automated algorithm [36]. We acquire more than 600 trajectories, all longer than 200 frames with some as long as 2000 frames, at a camera rate of 60 frame / s to allow the evaluation and the comparison of time- and ensemble-averaged MSD. The tMSD of individual trajectories displays a linear behavior $(\beta \sim 1)$, consistent with pure Brownian diffusion [Fig. 1(c)]. The fitting of the average tMSD provides a value $\beta=0.95 \pm 0.05$. In addition, the distribution of the 
(a)

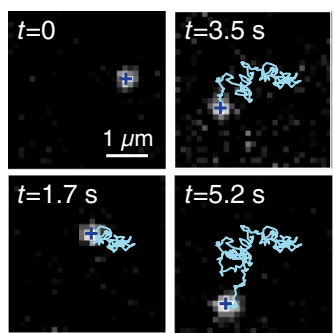

(c)

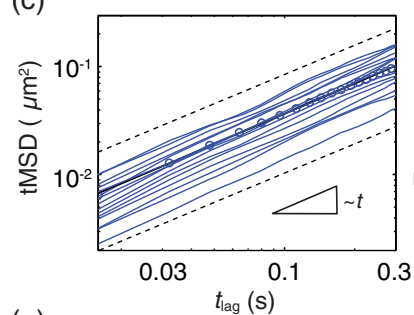

(e)

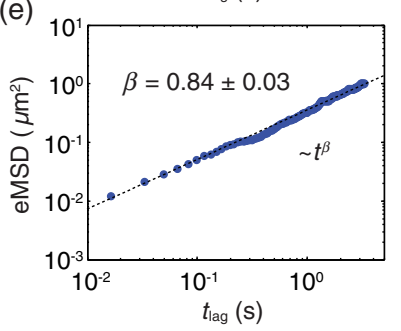

(b)

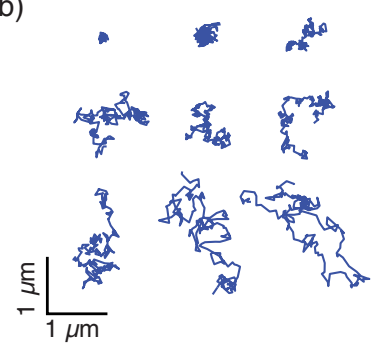

(d)

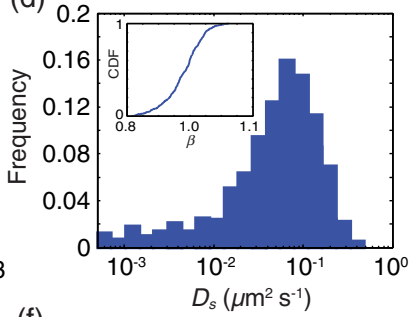

(f)

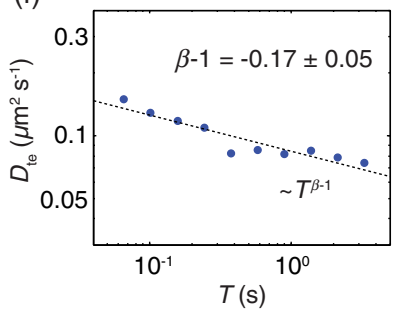

FIG. 1. DC-SIGN diffusion shows weak ergodicity breaking and aging. (a) Representative video frames of a quantum-dotlabeled WT DC-SIGN molecule diffusing on the dorsal membrane of a CHO cell. The centroid position of the bright spot (+), corresponding to a single quantum dot, is tracked and reconnected to build up the DC-SIGN trajectory, shown by the cyan line. (b) Representative trajectories for the same recording time (3.2s). (c) Log-log plot of the time-averaged MSD for individual trajectories (blue lines). The dashed lines scale linearly in time, showing that TMSD is compatible with pure Brownian motion $(\beta=1)$. The symbols (circle) correspond to the average tMSD. A linear fit to the log-log transformed data (black line) provides $\beta=0.95 \pm 0.05$. (d) Distribution of short-time diffusion coefficients as obtained from linear fitting of the time-averaged MSD for all the trajectories. Inset: Cumulative distribution function (CDF) of the exponent $\beta$ obtained from nonlinear fitting of the tMSD of all the trajectories. (e) Log-log plot of the ensembleaveraged MSD. A power-law fit of the data (dashed line) provides an exponent $\beta=0.84$, showing subdiffusion. (f) $\log -\log$ plot of the time-ensemble-averaged diffusion coefficient as a function of the observation time $T$. The diffusion coefficients are obtained by linear fitting of the time-ensemble-averaged MSD. A powerlaw fit (dashed line) provides an exponent $\beta-1=-0.17$, revealing aging and in good agreement with the value of $\beta$ found in (e).

exponents $\beta$ obtained by nonlinear fitting of the tMSDs of the individual trajectories [inset of Fig. 1(d)] shows an average $\langle\beta\rangle=0.98 \pm 0.06$.

Since the tMSD values corresponding to different trajectories are broadly scattered, for each trajectory, we calculate the diffusion coefficient $D_{s}$ by a linear fit of the

tMSD at time lags $<10 \%$ of the trajectory duration [37]. As expected, the resulting values of $D_{s}$ are found to have a very broad distribution, spanning more than 2 orders of magnitude [Fig. 1(d)].

However, in marked contrast with the tMSD, the eMSD deviates significantly from linearity, showing subdiffusion with an exponent $\beta=0.84 \pm 0.03$ [Fig. 1(e)]. The difference between the scalings of tMSD and eMSD is a clear signature of WEB [38]. To inquire whether DC-SIGN dynamics also exhibits aging, we compute the timeensemble-averaged MSD (teMSD) by truncating the data at different observation times $T$ :

$$
\begin{aligned}
\operatorname{teMSD}\left(t_{\text {lag }}, T\right)= & \frac{1}{J} \frac{1}{\frac{T}{\Delta t}-m} \\
& \times \sum_{i=1}^{(T / \Delta t)-m} \sum_{j=1}^{J}\left[x_{j}\left(t_{i}+m \Delta t\right)-x_{j}\left(t_{i}\right)\right]^{2}
\end{aligned}
$$

and extracting the corresponding diffusion coefficient $D_{\text {te }}$ by linear fitting [37]. In systems with uncorrelated increments, it can be shown under rather general assumptions that $D_{\text {te }} \sim T^{\beta-1}[21,39]$. The observed $D_{\text {te }}$ indeed scales as a power law with an exponent of $-0.17 \pm 0.05$ [Fig. 1(f)], yielding a value of $\beta$ in good agreement with the exponent determined from eMSD. These results thus demonstrate that WT DC-SIGN dynamics exhibits aging.

\section{FAILURE OF THE CTRW MODEL}

The motion of some biological components, including the Kv2.1 potassium channel in the plasma membrane [12], lipid granules in yeast cells [9], and insulin-containing vesicles in pancreatic $\beta$ cells [10], has been reported to exhibit subdiffusion compatible with the coexistence of an ergodic and a nonergodic process. The nonergodic part of the process has been modeled within the framework of the CTRW [28,38,39].

CTRW is a random walk in which a particle performs jumps whose lengths have a finite variance, but between jumps, the walker remains trapped for random dwell times,

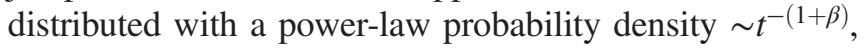
which for $\beta \leq 1$ has an infinite mean. The duration of trapping events is independent of the previous history of the system. The energy landscape of this process is characterized by potential wells with a broad depth distribution. Such energetic disorder yields nonergodicity, since no matter how long one measures, deep traps cause dwell times on the order of the measurement time. Within the biological context, these traps have generally been associated with chemical binding to stationary cellular components (e.g., the actin cytoskeleton [12] or microtubules [10]), with a distribution of dissociation times with varying lifetimes. tMSDs of molecules performing CTRW show broadly scattered values but are, on average, linear in the 
lag time $t_{\text {lag }}[39,40]$, similar to our observation in Fig. 1(c). The subdiffusive behavior of the motion emerges in the eMSD, scaling with the same exponent $\beta$ describing the probability density of trapping dwell times.

Since DC-SIGN dynamics also show nonergodic subdiffusion and aging, we seek to investigate whether DC-SIGN diffusion agrees with the predictions of the CTRW model. To this end, we search for the occurrence of transient trapping events on individual trajectories.

In SPT experiments, the limited localization accuracy for determining the particle position sets a lower limit for the diffusivity value that can experimentally be measured. In our case, this lower threshold lies at $D_{\text {th }}=6 \times 10^{-4} \mu \mathrm{m}^{2} \mathrm{~s}^{-1}$. Therefore, a segmentation algorithm [41] is applied to the $x$ and $y$ displacements of our trajectories in order to detect events with diffusivity lower than $D_{\text {th }}$. Surprisingly, transient trapping is only detected over less than $5 \%$ of the total recording time [Figs. 2(a)-2(c)]. The detected trapping times display an average duration of $330 \pm 30 \mathrm{~ms}$ [Fig. 2(d)]. An alternative analysis, based on the transient confinement zone algorithm [42], gave comparable results [43].

In order to understand the nature of these trapping events, we attempt to fit their distribution by means of both an exponential and a power-law function $\sim t^{-(1+\beta)}$, as expected for CTRW [12]. The power-law probability density function provides a better fit to the data, yielding an exponent $\beta=0.83 \pm 0.05$ [Fig. 2(d)], in agreement with the value obtained for the eMSD. While a power-law distribution of trapping-event durations would be compatible with the behavior expected for the CTRW, it is unlikely that these trapping events can have a major role in the ergodicity breaking we observe, given their very small probability of occurrence. In addition, we notice here that various other models predict a similar scaling of the trapping times; an example will be discussed in detail in Sec. IV. To quantify to what extent the small percentage of trapping events actually influences the nonergodic behavior, we calculate the eMSD excluding completely the trajectories showing events compatible with immobilization. Interestingly, this analysis provides an exponent $\beta=$ $0.84 \pm 0.04$ exactly coinciding with the value obtained for the full set of trajectories [Fig. 2(d)], thus confirming that trapping alone cannot account for the ergodicity breaking we observe in WT DC-SIGN dynamics.

In addition, we construct the distribution of escape times by identifying the duration of the events in which a trajectory remains within a given radius $R_{\mathrm{TH}}$ [Fig. 2(e)]. For a CTRW, the long-time dynamics is dominated by anomalous trapping events, and, as a result, this quantity is expected to be independent of $R_{\mathrm{TH}}$ [12]. In strong contrast to the CTRW model, the escape-time distributions of DC-SIGN trajectories show a marked dependence on $R_{\mathrm{TH}}$ [Fig. 2(f)].

In summary, the rare occurrence of transient trapping events, the dependence of escape-time distributions on $R_{\mathrm{TH}}$, and, most importantly, the fact that tMSD and eMSD (a)
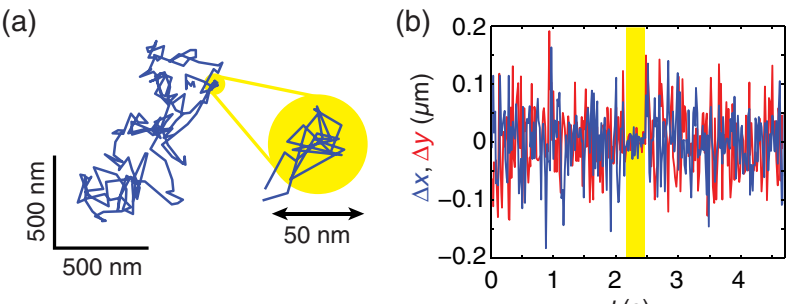

(c)

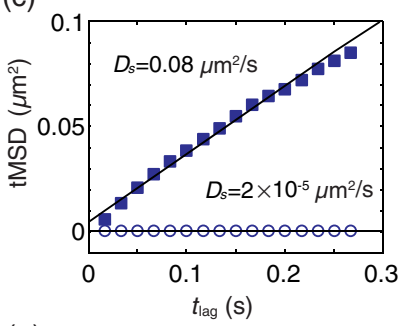

(d)

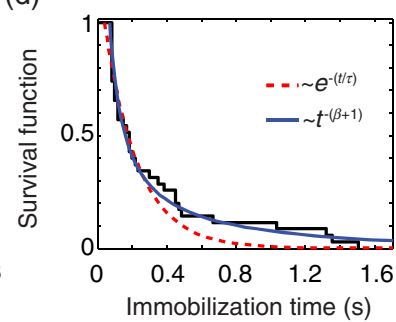

(e)

(f)
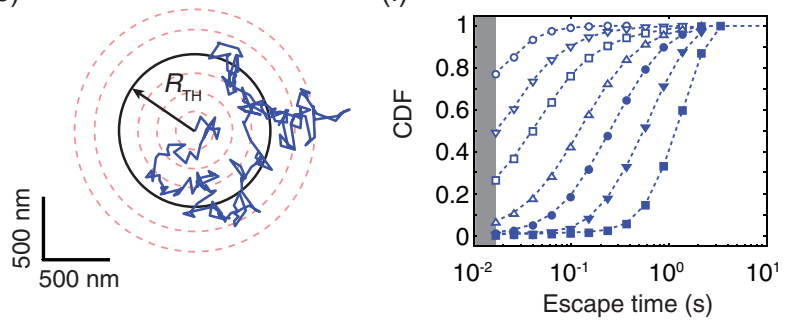

FIG. 2. DC-SIGN receptor dynamics is inconsistent with the CTRW model. (a) A trajectory of WT DC-SIGN on living-cell membranes showing a short-lived transient immobilization event, highlighted by the yellow circular area. (b) Plot of the $x$ (blue) and $y$ (red) displacements as a function of time. The occurrence of transient immobilization (yellow region) corresponds to a reduction in the trajectory displacement. (c) Time-averaged MSD for the entire trajectory (filled square) and for the immobilization region only (open circle). (d) Survival function of the duration of immobilization events for WT DC-SIGN trajectories (black line). Red and blue lines correspond to fits to exponential and powerlaw distribution functions, respectively. The power-law fit provides $\beta=0.83 \pm 0.05$, in agreement with the exponent obtained for the eMSD. (e) Schematic representation of the calculation of the escape-time probability from circular areas of different radius $R_{\mathrm{TH}}$. (f) CDF of the trajectory escape time for different radii $R_{\mathrm{TH}}=20$ (open circle), 50 (open inverted triangle), 100 (open square), 200 (open triangle), 300 (filled circle), 500 (filled inverted triangle), and 1000 (filled square) $\mathrm{nm}$. Dashed lines are guides to the eye. The gray-shaded region represents times shorter than the acquisition frame rate.

show different scaling even when the few trajectories showing immobilization are removed from the analysis are all inconsistent with CTRW, indicating that the main features of the DC-SIGN dynamics may not be explained in terms of this model.

\section{DC-SIGN DISPLAY CHANGES OF DIFFUSIVITY}

Recently, diffusion maps of the cell membrane have shown the presence of patches with strongly varying 
diffusivity $[36,44,45]$. Based on this evidence, we have recently proposed a class of models describing ordinary Brownian motion with a diffusivity that varies randomly but is constant on time intervals or spatial patches with random size [21]. These models describe anomalous diffusion and WEB in complex and heterogeneous media, such as the cellular environment, without invoking transient trapping.

To address whether the observed nonergodic dynamics of DC-SIGN can be described with this theoretical framework, we further analyze individual trajectories by means of a change-point algorithm to detect variations of diffusivity in time [41]. In brief, the algorithm consists in a likelihood-based approach to quantitatively recover timedependent changes in diffusivity, based on the calculation of maximum-likelihood estimators for the determination of diffusion coefficients and the application of a likelihoodratio test for the localization of the changes. Notably, DC-SIGN trajectories display a Brownian motion with relatively constant diffusivity over intervals of varying length but which changes significantly between these (a)

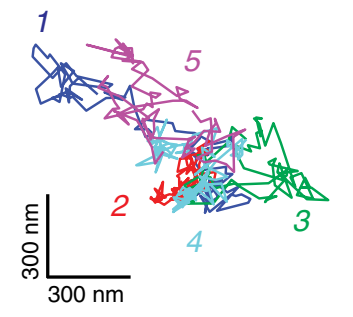

(c)

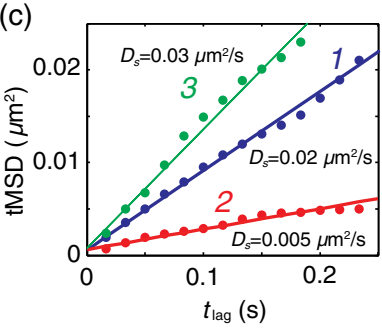

(b)

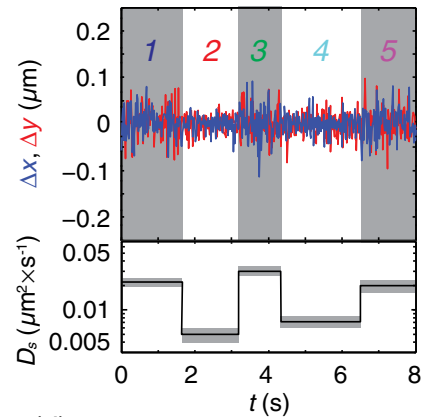

(d)

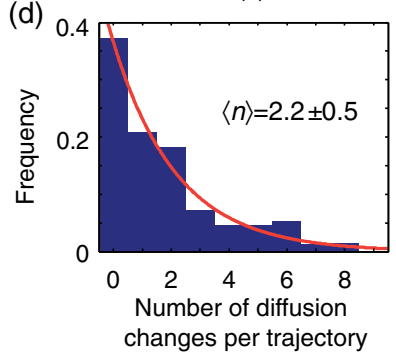

FIG. 3. DC-SIGN motion experiences changes in diffusivity. (a) Representative WT DC-SIGN trajectory displaying changes of diffusivity. Change-point analysis evidences five different regions represented with different colors. (b) Plot of the $x$ (blue) and $y$ (red) displacements for the trajectory in (a) as a function of time. The shaded areas indicate the regions of different diffusivity. The lower panel displays the corresponding short-time diffusion coefficient as obtained from a linear fit of the timeaveraged MSD for the five different regions. Gray areas correspond to the 95\% confidence level. (c) Plot of time-averaged MSD versus time lag for the first three regions of the trajectory in (a). (d) Histogram of the number of changes of diffusion per trajectory. Most of the trajectories (63\%) display at least one dynamical change, with an average of 2.2 changes per trajectory. intervals [Figs. 3(a)-3(c)]. Similar features are identified in a large fraction of trajectories, with $63 \%$ showing at least one diffusivity change [Fig. 3(d)], in qualitative agreement with the models of random diffusivity [21].

To obtain a comprehensive understanding of our data, we consider an annealed model in which randomly diffusing particles undergo sudden changes of diffusion coefficient [21]. The distribution of diffusion coefficients $D$ that a particle can experience is assumed to have a power-law behavior $\sim D^{\sigma-1}$ for small $D$ (with $\sigma>0$ ) and a fast decay for $D \rightarrow \infty$. Given $D$, the transit time $\tau$ (i.e., the time $\tau$ a particle moves with a given $D$ ) is taken to have a probability distribution with a mean $\sim D^{-\gamma}$ (with $-\infty<\gamma<\infty$ ). Since the motion during the transit time $\tau$ is Brownian, particles explore areas with radius $r \sim \sqrt{\tau D}$, and the radius of the region explored with such a diffusion coefficient has a probability distribution with a mean $\sim D^{(1-\gamma) / 2}$. Depending on the values of the exponents $\sigma$ and $\gamma$, this model predicts three regimes [21], which are, namely, the following. (0) For $\gamma<\sigma$, the long-time dynamics is compatible with ordinary Brownian motion and yields an eMSD exponent $\beta=1$; (I) for $\sigma<\gamma<\sigma+1$, the average transit time $\tau$ diverges and particles undergo nonergodic subdiffusion with $\beta=\sigma / \gamma$; and (II) for $\gamma>\sigma+1$, both the average transit time $\tau$ and the average of the radius squared $r^{2}$ of the explored area diverge and one obtains nonergodic subdiffusion with $\beta=1-1 / \gamma$. On the other hand, the tMSD predicted by this model remains linear in time for $t \ll T$, for every choice of $\sigma$ and $\gamma$.

We perform in silico experiments of 2D diffusion [Figs. 4(a) and 4(b)], assuming a distribution of diffusion coefficients $D$ given by

$$
P_{D}(D)=\frac{D^{\sigma-1} e^{-D / b}}{b^{\sigma} \Gamma(\sigma)}
$$

and a conditional distribution of transit times $\tau$ given by

$$
P_{\tau}(\tau \mid D)=\frac{D^{\gamma}}{k} e^{-\tau D^{\gamma} / k}
$$

where $b$ and $k$ are dimensional constants and $\Gamma(x)$ is the Gamma function.

The functional forms of the distributions in Eqs. (4) and (5) comply with the requirements of our model, while at the same time, they ensure the minimal number of free parameters, making them a natural choice for our theoretical analysis. However, we note here that the asymptotic behavior of the model is actually independent of the specific functional form of the joint distributions. We perform simulations for different values of $\sigma$, with $\gamma=\sigma / \beta$ as in regime (I), and $\beta=0.84$, the exponent obtained from the experimental eMSD. The simulations quantitatively reproduce not only subdiffusion, nonergodicity, and aging but also the heterogeneous distribution 

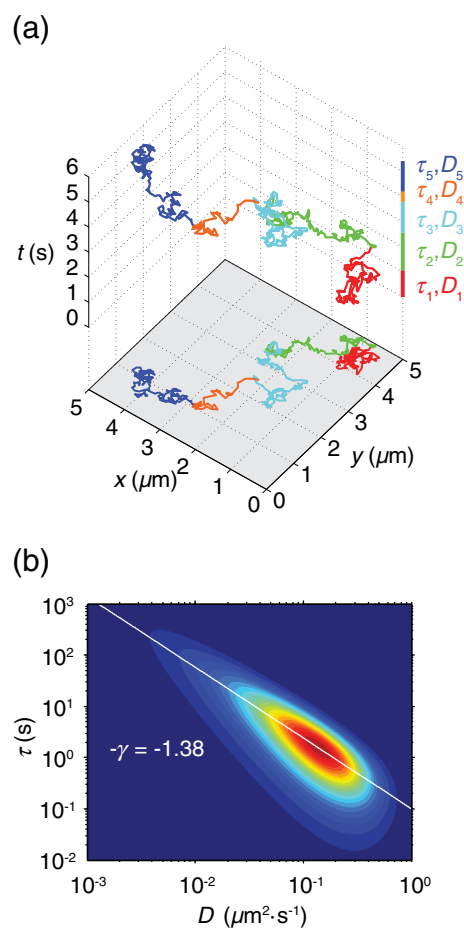

(c)



(d)

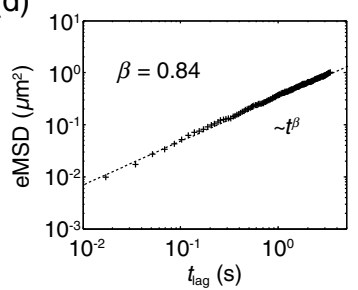

(e)

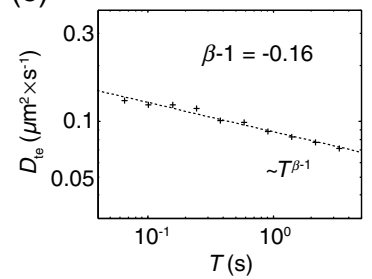

(f)

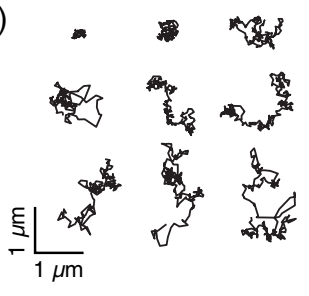

(g)

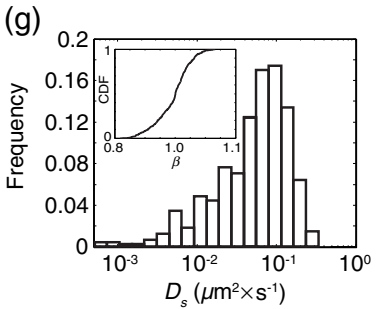

(h)

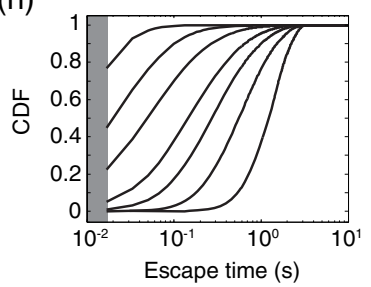

FIG. 4. Annealed model of heterogeneous diffusion quantitatively reproduces DC-SIGN motion. (a) A simulated trajectory composed by five time intervals with different transit times $\tau_{i}$ and diffusivity $D_{i}$. (b) Contour plot of the probability distribution of the simulated diffusion coefficient $D$ and transit time $\tau$ for the parameters reproducing the dynamics of WT DC-SIGN $(\sigma=1.16, \gamma=1.38$, $\left.b=0.12 \mu \mathrm{m}^{2} / \mathrm{s}, k=0.10 \mu \mathrm{m}^{2 \gamma} \mathrm{s}^{\gamma+1}\right)$. The white line represents the power-law dependence between diffusivity and average transit time with exponent $-\gamma$. (c) Log-log plot of the time-averaged MSD for simulated trajectories (black lines). The symbols (circle) correspond to the average tMSD. The linear fit to the $\log -\log$ transformed data provides $\beta=0.98 \pm 0.03$. (d) $\log -\log$ plot of the ensemble-averaged MSD for the simulated trajectories. The dashed line represents a power law with the theoretical exponent $\beta=\sigma / \gamma=0.84$. (e) Log-log plot of the time-ensemble-averaged diffusion coefficient as a function of the observation time $T$. The dashed line represents a power law with the theoretical exponent $\beta-1=-0.16$. (f) Simulated trajectories for the same recording time (3.2s). (g) Distribution of short-time diffusion coefficients as obtained from linear fitting of the time-averaged MSD for all the simulated trajectories. Inset: CDF of the exponent $\beta$ obtained from nonlinear fitting of the tMSD of all the trajectories. (h) CDF of trajectory escape time for different radii. Curves from left to right correspond to radii $R_{\mathrm{TH}}=20,50,100,200,300,500$, and $1000 \mathrm{~nm}$.

of diffusion coefficients and escape-time distributions [Figs. 4(c)-4(h)]. The remarkable agreement between simulations and experimental data strongly supports heterogeneous diffusion as the origin for DC-SIGN nonergodicity.

It must be noticed that, in contrast to CTRW, our model does not assume particle immobilization but a continuous distribution of diffusivity, with $P_{D}(D) \sim D^{\sigma-1}$ for small $D$. However, from the experimental point of view, it is not possible to distinguish immobilization from very slow diffusion. In fact, the limited localization accuracy of SPT experiments translates into a lower limit for the diffusivity value $D_{\text {th }}$ that can be detected. Therefore, in our analysis, trajectories, or a portion of trajectories, with diffusivity lower than this threshold value $\left(D_{\mathrm{th}}=6 \times 10^{-4} \mu \mathrm{m}^{2} \mathrm{~s}^{-1}\right)$ are identified as immobile, as shown in Figs. 2(a) and 2(b). From the model described above, the distribution of the duration of these "apparent" immobilizations can be calculated as

$$
P_{\mathrm{imm}}(\tau)=\int_{0}^{D_{\mathrm{th}}} P_{D}(D) P_{\tau}(\tau \mid D) d D
$$

We neglect here the possibility that the trajectory of an in silico particle contains two consecutive segments characterized by diffusivities $D_{i}$ and $D_{i+1}$, which are both smaller than $D_{\text {th }}$, as this probability is vanishingly small for the parameter regime of our setup. Independently of $D_{\text {th }}$, the integral in Eq. (6) scales asymptotically as $\tau^{-1-\beta}$ with $\beta=(\sigma / \gamma)$, providing for the distribution of immobilization events the same behavior predicted by the CTRW [12]. Therefore, the distribution of immobilization times in Fig. 2(d) is fully compatible with the prediction of our model, further confirming its agreement with the experimental data.

\section{DYNAMICS OF RECEPTOR MUTANTS}

From the structural point of view [Fig. 5(a)], DC-SIGN is a tetrameric transmembrane protein, with each of the four 

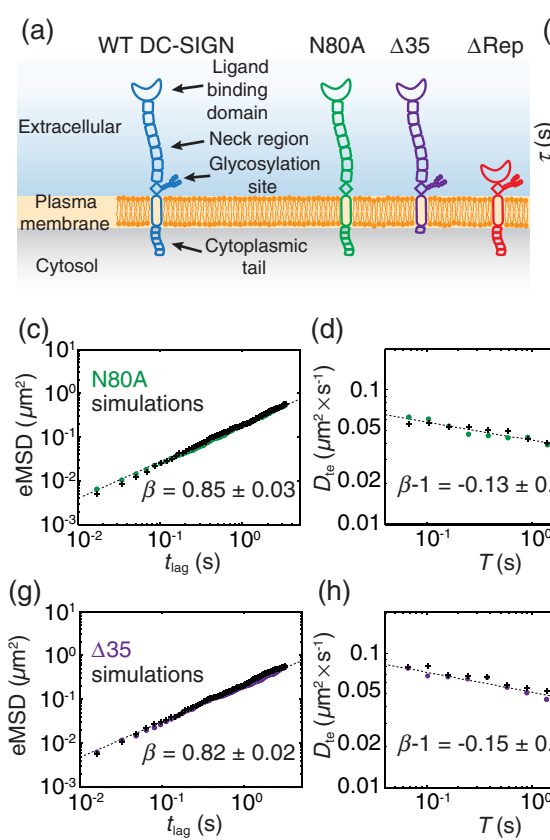

(d)

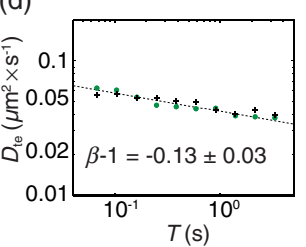

(h)


(n)

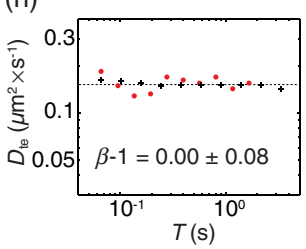

(b)

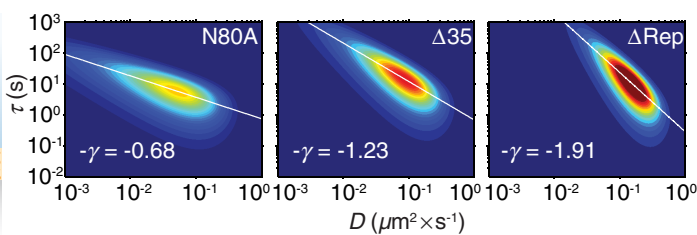

(e)

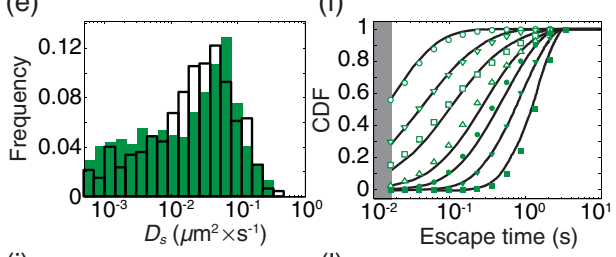

(i)

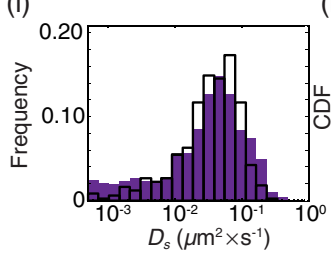

(l)

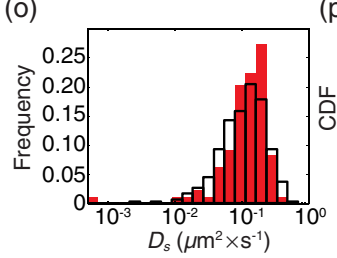

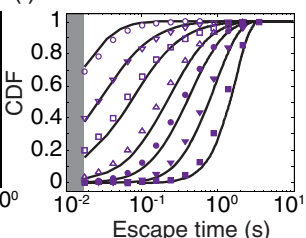

(p)

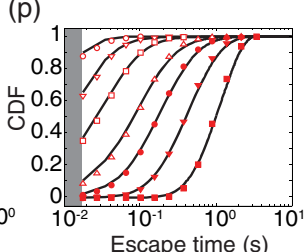

FIG. 5. Effect of mutations on the dynamics of DC-SIGN. (a) Schematic representation of WT DC-SIGN and its mutated forms. (b) Contour plot of the probability distribution of the simulated diffusion coefficient $(D)$ and transit time $(\tau)$ for the parameters reproducing the dynamics of $\mathrm{N} 80 \mathrm{~A}\left(\sigma=0.58, \gamma=0.68, b=0.09 \mu \mathrm{m}^{2} / \mathrm{s}, k=0.74 \mu \mathrm{m}^{2 \gamma} \mathrm{s}^{\gamma+1}\right), \Delta 35(\sigma=1.04, \gamma=1.23$, $\left.b=0.08 \mu \mathrm{m}^{2} / \mathrm{s}, k=0.07 \mu \mathrm{m}^{2 \gamma} \mathrm{s}^{\gamma+1}\right)$, and $\Delta \operatorname{Rep}\left(\sigma=2.11, \gamma=1.91, b=0.07 \mu \mathrm{m}^{2} / \mathrm{s}, k=0.07 \mu \mathrm{m}^{2 \gamma} \mathrm{s}^{\gamma+1}\right)$. The white line represents the power-law dependence between diffusivity and average transit time with exponent $-\gamma$. (c) Log-log plot of the ensemble-averaged MSD for N80A trajectories (filled circle) and simulated data (+). (d) Log-log plot of the time-ensemble-averaged diffusion coefficient for N80 A trajectories (filled circle) and simulated data $(+)$ as a function of the observation time $T$. (e) Distribution of short-time diffusion coefficients as obtained from linear fitting of the time-averaged MSD for the N80A (filled bars) and the simulated trajectories (empty bars). (f) CDF of the escape time for N80A (symbols) and simulated trajectories (lines) for different radii. The meaning of the symbols is the same as in Fig. 2(f). (g)-(l) Dynamical behavior of the $\Delta 35$ mutant. (m)-(p) Dynamical behavior of the $\Delta$ Rep mutant.

subunits comprising (i) an extracellular domain that allows binding of the receptor to pathogens, i.e., a ligand-binding domain; (ii) a long-neck region; and (iii) and a transmembrane part followed by a cytoplasmic tail that allows interactions with inner cell components and facilitates the uptake and internalization of pathogens [46,47]. Moreover, DC-SIGN contains a single $N$-glycosylation site mediating interactions with glycan-binding proteins [43]. To gain insight into the molecular mechanisms of DC-SIGN nonergodic diffusion, we generate three mutated forms of the receptor [Fig. 5(a)]. These mutations have been reported to modify the interaction of DC-SIGN with other cellular components, strongly affecting DC-SIGN function $[43,48,49]$. The N80A mutant lacks the $N$-glycosylation site. This defect hinders interactions of DC-SIGN with components of the extracellular membrane that bind to sugars [43]. The $\Delta 35$ mutant lacks a significant part of the cytoplasmic tail, preventing interactions with cytosolic components such as actin [48]. Finally, the $\Delta$ Rep mutant lacks part of the neck region, abrogating interactions between different DC-SIGN molecules [49].

We find that each mutation has a very different effect on the dynamics of the receptor. The N80A mutant [Fig. 5(c)-5(f)] shows nonergodic subdiffusion, with an exponent $\beta$ similar to the one measured for WT DC-SIGN. However, N80A shows a significantly larger extent of heterogeneity in the diffusion-coefficient distribution, with a lower median diffusivity. The $\Delta 35$ mutant [Figs. 5(g)-5(l)] also shows nonergodic subdiffusion. The anomalous exponent and the distribution of the diffusion coefficients are similar to that of WT DCSIGN, with only a slight reduction in median diffusivity. We accurately reproduce N80A and $\Delta 35$ dynamics by simulations performed in regime (I), i.e., nonergodic 
subdiffusion, using comparable values of $\gamma$ for WT DC-SIGN and $\Delta 35$, and a smaller value of $\gamma$ for N80A [Fig. 5(b)]. On the other hand, $\Delta$ Rep dynamics yield ergodic Brownian diffusion [Figs. 5(m)-5(p)] and a narrower distribution of diffusivity with the median value significantly higher than for WT DC-SIGN. Consistently, the behavior of $\Delta$ Rep is fully captured by in silico experiments in regime (0), i.e., ordinary Brownian motion.

Overall, these results demonstrate that the molecular structure of the receptor strongly influences its diffusive behavior on the cell membrane and the occurrence of weak ergodicity breaking.

\section{NONERGODICITY AND BIOLOGICAL FUNCTION}

Together with our previous biophysical studies on DC-SIGN $[43,49]$, the data and analysis presented in this paper allow us to link the dynamical behavior of DC-SIGN to its functional role in pathogen capture and uptake (known as endocytosis).

In terms of steady-state organization, WT DC-SIGN, $\mathrm{N} 80 \mathrm{~A}$, and $\Delta 35$ preferentially form nanoclusters on the cell membrane, which are crucial for regulating pathogen binding [43,49], whereas removal of the neck region $(\Delta$ Rep) reduces nanoclustering and binding efficiency to small pathogens, such as viruses [49]. Our results thus show that the diffusive behavior of the receptor is strongly linked to nanoclustering but not merely due to sizedependent diffusivity and/or time-dependent cluster formation and breakdown. In fact, dual-color SPT experiments performed at high labeling density do not reveal correlated motion between nearby DC-SIGN nanoclusters, excluding the occurrence of dynamic nanocluster coalescence [43]. Moreover, although superresolution imaging has revealed that WT DC-SIGN, N80A, and $\Delta 35$ form nanoclusters with similar distributions of size and stoichiometry [43,49], our dynamical data evidence significant differences in their diffusion patterns (Figs. 1 and 5).

Our data are, in fact, consistent with the view of the plasma membrane as a highly dynamic and heterogeneous medium, where WEB stems from the enhanced ability of DC-SIGN nanoclusters to interact with the membrane environment, including components from the outer and inner membrane leaflet. This interaction is inhibited (or strongly reduced) in the case of the $\Delta$ Rep mutant since it does not form nanoclusters [49]. As a result, the motion of $\Delta$ Rep is Brownian and ergodic, and, interestingly, this dynamic behavior correlates with its impaired pathogenbinding capability [34,49].

In contrast, we observe that both WT DC-SIGN and N80A, which show a similar degree of nanoclustering [43], exhibit WEB. But, the distribution of diffusivity of N80A is significantly broader than that of WT DC-SIGN and is shifted toward lower diffusivity values [Figs. 5(c)-5(f)]. This increased heterogeneity correlates with altered interactions of the N80A with extracellular components, resulting from the removal of the glycosylation site. Indeed, we have recently shown that the N80A mutant has a reduced capability to interact with extracellular sugarbinding partners [43]. Thus, it appears that the extracellular milieu next to the membrane contributes to the degree of dynamical heterogeneity sensed by the receptor. Remarkably, this correlation also extends to the functional level, as we have recently shown that interactions of DCSIGN with extracellular sugar-binding proteins influence encounters of DC-SIGN with the main endocytic protein clathrin. In turn, this altered interaction with clathrin results in reduced clathrin-dependent endocytosis of the receptor and its pathogenic ligands [43].

Finally, the $\Delta 35$ mutant exhibits nanoclustering [49] and WEB similar to that of WT DC-SIGN. From the biological point of view, however, this mutant is not able to interact with cytosolic components in close proximity to the inner membrane leaflet, including actin [48]. Therefore, in contrast to the extracellular influence observed for the N80A mutant, the results obtained for the $\Delta 35$ mutant indicate that interactions with the actin cytoskeleton, responsible for the CTRW-like behavior of other proteins [12], do not play a major role in DC-SIGN WEB. Nevertheless, it should be mentioned that the reduced endocytic capability of the $\Delta 35$ could not be uniquely attributed to its dynamic behavior on the cell membrane but rather to its impaired interaction with downstream partners involved in internalization $[48,49]$.

\section{CONCLUSIONS AND OUTLOOK}

We have demonstrated that the DC-SIGN receptor displays subdiffusive dynamics, characterized by weak ergodicity breaking and aging. In contrast to other biological systems, receptor trajectories do not show significant evidence of transient immobilization with power-lawdistributed waiting times. Therefore, its nonergodic behavior cannot be explained in terms of the CTRW model. However, DC-SIGN dynamics is highly heterogeneous, with trajectories often displaying sudden changes of diffusivity. These features are accurately described by a novel model of ordinary diffusion in complex media, strongly suggesting inhomogeneous diffusivity as the cause of DC-SIGN nonergodic behavior. Comparative analysis of three mutated forms of DC-SIGN evidences the importance of specific regions of the receptor structure, known to mediate interactions with other molecules, in receptor dynamics. Since the mutations of these regions differently impair receptor function, the experiments allowed us to establish the relevance of nonergodicity for the regulation of functional mechanisms, such as the capacity for pathogen recognition and internalization.

The evidence that temporal and/or spatial disorder induces subdiffusion and WEB agrees remarkably well with the current view of the plasma membrane as an extremely complex environment. Here, precise tuning of 
the spatiotemporal organization of membrane components, in addition to biochemical interactions with molecules in the inner and outer membrane leaflet, orchestrates the triggering of cell-signaling pathways. A detailed understanding of how these specific interactions occur and affect dynamics is still lacking. Future experiments, involving simultaneous tracking of several proteins by means of multicolor SPT [44], might provide a deeper comprehension of these mechanisms at the molecular level.

The model used to interpret our data provides a flexible and realistic framework to describe anomalous motion in cell membranes. Although in the present work we have focused our simulations on time-dependent changes of diffusivity, similar conclusions can be obtained assuming a spatial dependence, with constant diffusivity on membrane patches of random size [21]. The current data do not allow discrimination between the two scenarios. The application of techniques that combine dynamic and spatial mapping at high labeling conditions $[45,50]$ would be necessary to verify the occurrence of spatial maps of diffusivity. In addition, numerical simulations of spatial-dependent random diffusivity require the construction of two-dimensional diffusivity maps consistent with the model's probability densities, which is a nontrivial task.

While the work presented here focuses on the cell plasma membrane, we point out that these results have much broader implications. In fact, our model and analysis are very general and can be applied to any diffusive system that shows WEB, in order to investigate the role that heterogeneous diffusivity plays in observed anomalies. Fundamental questions about the nature of anomalous and nonergodic diffusion in disordered media arise in many fields, such as life sciences [28], soft condensed matter [51,52], ultracold gases [53,54], geology [55], and ecology [56]. Our work provides an alternative conceptual framework and specific tools for answering these questions.

\section{ACKNOWLEDGMENTS}

We thank A. Cambi for providing DC-SIGN transfected $\mathrm{CHO}$ cells, O. Esteban for preparing the Fab fragments, P. Symeonidou Besi for preliminary data analysis, and B. Castro for recording the N80A trajectories. This work was supported by Fundació Cellex, Generalitat de Catalunya (Grant No. 2009 SGR 597), the European Commission (FP7-ICT-2011-7, Grant No. 288263), the HFSP (Grant No. RGP0027/2012), ERC AdG Osyris, and the Spanish Ministry of Science and Innovation (Grants FOQUS and No. MAT2011-22887).

C. M. and J. A. T.-P. contributed equally to this work.

\section{APPENDIX A: CELL CULTURE AND LABELING}

CHO cells stably transfected with DC-SIGN mutants are cultured in HAM's F-12 medium (LabClinics) supplemented with $10 \%$ fetal calf serum and antibiotic or antimycotic (Gibco). CHO cells are seeded onto $25-\mathrm{mm}$ cover slips $24 \mathrm{~h}$ before imaging. Streptavidin-coated quantum dots (Qdot655, Invitrogen) are added to an equimolar solution of biotinylated anti-DC-SIGN DCN46 Fab fragment (or anti-AU1 monovalent Ab, in the case of a $\Delta$ Rep mutant) and a $50 \times$ excess of free biotin (Gibco) in order to obtain a 1:1 Fab fragment-quantum-dot ratio (or a monovalent Ab-quantum-dot ratio, in the case of a $\Delta$ Rep mutant). In these conditions, we estimate a $0.04 \%$ probability of having multiple Fab fragments or Abs bound to the same quantum dot. Cells are incubated for $5 \mathrm{~min}$ at room temperature with 50-pM conjugated quantum dots in a cold phosphate-buffered saline buffer supplemented with $6 \%$ bovine serum albumin. Extensive washing is performed to remove nonbound conjugated quantum dots before imaging. In parallel with each experiment, we also perform control experiments by labeling DC-SIGN-negative $\mathrm{CHO}$ cells. The lack of quantum dots binding to the DC-SIGN-negative $\mathrm{CHO}$ cells confirms the absence of nonspecific binding. Furthermore, the low-concentration labeling conditions are chosen to minimize the probability of having more than one quantum dot within the same region of interest. Fab fragments and monovalent $\mathrm{Ab}$ are obtained as described in Refs. [43,49].

\section{APPENDIX B: SINGLE-PARTICLE-TRACKING EXPERIMENTS}

We perform video microscopy using a custom singlemolecule sensitive epifluorescence microscope. Continuous excitation is provided by the 488-nm line of an argonion laser (Spectra Physics), with power density at the sample plane of approximately $0.3 \mathrm{~kW} / \mathrm{cm}^{2}$. Fluorescence is collected by means of a 1.2-numerical aperture waterimmersion objective (Olympus) and guided into an intensified electron multiplied CCD camera (Hamamatsu) after suitable filtering. Movies are recorded on the dorsal membrane of $\mathrm{CHO}$ cells at a $60-\mathrm{Hz}$ frame rate. Experiments are performed in a culture dish incubator (DH-35iL, Warner Instrument) equipped with a temperature controller (TC-324B, Warner Instrument) and a digital $\mathrm{CO}_{2}$ controller (DGTCO2BX, Okolab) at $37^{\circ} \mathrm{C}$ and in a $5 \% \mathrm{CO}_{2}$ atmosphere. Trajectories are analyzed with a custom Matlab code based on the algorithm described in Ref. [36]. In order to avoid artifacts in trajectory reconnection caused by quantum-dot blinking dynamics and/or high local density of quantum dots, each trajectory is terminated at the first video frame not displaying a clearly identifiable bright spot in the surrounding of the quantum-dot localization obtained in the previous frame. Similarly, the trajectory reconstruction is also interrupted if the presence of multiple bright spots does not allow unambiguous identification of the same quantum dots in successive video frames. As a further check for false-positive reconnection, trajectories are overlaid to raw movies and visually inspected. 


\section{APPENDIX C: DATA ANALYSIS}

Time-, ensemble-, and time-ensemble-averaged meansquared displacements are calculated as described in Ref. [12]. Exponents of the eMSD, average tMSD, and $D_{\mathrm{t}, \text { ens }}$ are obtained by linear fitting of the log-log transformed data. Errors are calculated as the $99 \%$ confidence interval of the fitting parameters. Short-time diffusion coefficients are extracted from the linear fit of the first $10 \%$ of the points of tMSD curves [37].

Measurements of the apparent diffusion of quantum dots on fixed cells and glass cover slips are used to estimate the smallest detectable diffusivity. Short-time diffusion coefficients are obtained as described above for trajectories of immobilized quantum dots, and the corresponding probability distribution is calculated. $95 \%$ of the immobilized quantum-dot trajectories show values lower than $6 \times 10^{-4} \mu \mathrm{m}^{2} / \mathrm{s}$, which is therefore set as the threshold $\left(D_{\text {th }}\right)$ for classifying a trajectory as mobile.

Dynamical changes in the motion of DC-SIGN receptors are identified by application of the change-point algorithm described in Ref. [41]. In brief, the trajectories are recursively segmented and a maximum-likelihood-ratio test is applied to the trajectory displacements $(\Delta x, \Delta y)$ in order to identify sudden changes of diffusivity. The critical values for type-I error rates are set to a confidence level of $99 \%$, corresponding to $1 \%$ probability of having a false-positive identification of a change point. For each dynamical region identified by the algorithm, the short-time diffusion coefficient is calculated from a linear fit of the first $10 \%$ of the points of the corresponding MSD curves [37]. Regions showing a short-time diffusion coefficient lower than $D_{\text {th }}$ are considered compatible with transient immobilization.

\section{APPENDIX D: SIMULATIONS}

Simulated trajectories (500 per parameter set) are obtained by generating random diffusion coefficients $D$ according to the probability distribution given in Eq. (4). For each diffusion coefficient, the corresponding transit time $\tau$ is calculated as a random number drawn from the distribution given in Eq. (5). Particle coordinates $\mathbf{r}=\{x, y\}$ are generated as

$$
\mathbf{r}_{t+\Delta t^{\prime}}=\mathbf{r}_{t}+\sqrt{2 D \Delta t^{\prime}} \boldsymbol{\xi},
$$

where $\xi=\left\{\xi_{x}, \xi_{y}\right\}$ are pairs of random numbers from a Gaussian distribution with zero mean and unitary standard deviation. The time increment is calculated as $\Delta t^{\prime}=\Delta t / n$, where $\Delta t$ is the camera-acquisition rate and $n$ is an integer depending on $D$ and $\tau$, chosen in order to have at least ten points for each interval. For comparison with SPT data, trajectories are subsampled at the camera-acquisition rate. Simulated trajectories are generated with duration $T_{\text {sim }} \geq 3 T_{\exp }$, where $T_{\exp }$ is the duration of the experimental trajectory. The starting point is randomly drawn from a uniform distribution defined within 0 and $T_{\text {sim }}-T_{\text {exp }}$. Trajectories are then cut to have the same duration $T_{\exp }$ as the experimental ones. Gaussian noise corresponding to the experimental localization accuracy $\left(\sigma_{\text {acc }}=20 \mathrm{~nm}\right)$ is subsequently added to the trajectories.

[1] S. Condamin, O. Bénichou, V. Tejedor, R. Voituriez, and J. Klafter, First-Passage Times in Complex Scale-Invariant Media, Nature (London) 450, 77 (2007).

[2] M. A. Lomholt, I. M. Zaid, and R. Metzler, Subdiffusion and Weak Ergodicity Breaking in the Presence of a Reactive Boundary, Phys. Rev. Lett. 98, 200603 (2007).

[3] S. Condamin, V. Tejedor, R. Voituriez, O. Bénichou, and J. Klafter, Probing Microscopic Origins of Confined Subdiffusion by First-Passage Observables, Proc. Natl. Acad. Sci. U.S.A. 105, 5675 (2008).

[4] D. Choquet and A. Triller, The Role of Receptor Diffusion in the Organization of the Postsynaptic Membrane, Nat. Rev. Neurosci. 4, 251 (2003).

[5] A. Kusumi, T. a. Tsunoyama, K. M. Hirosawa, R. S. Kasai, and T. K. Fujiwara, Tracking Single Molecules at Work in Living Cells, Nat. Chem. Biol. 10, 524 (2014).

[6] I. Bronstein, Y. Israel, E. Kepten, S. Mai, Y. Shav-Tal, E. Barkai, and Y. Garini, Transient Anomalous Diffusion of Telomeres in the Nucleus of Mammalian Cells, Phys. Rev. Lett. 103, 018102 (2009).

[7] M. Weiss, M. Elsner, F. Kartberg, and T. Nilsson, Anomalous Subdiffusion Is a Measure for Cytoplasmic Crowding in Living Cells, Biophys. J. 87, 3518 (2004).

[8] I. Golding and E. C. Cox, Physical Nature of Bacterial Cytoplasm, Phys. Rev. Lett. 96, 098102 (2006).

[9] J.-H. Jeon, V. Tejedor, S. Burov, E. Barkai, C. SelhuberUnkel, K. Berg-Sørensen, L. Oddershede, and R. Metzler, In Vivo Anomalous Diffusion and Weak Ergodicity Breaking of Lipid Granules, Phys. Rev. Lett. 106, 048103 (2011).

[10] S. M. A. Tabei, S. Burov, H. Y. Kim, A. Kuznetsov, T. Huynh, J. Jureller, L. H. Philipson, A. R. Dinner, and N. F. Scherer, Intracellular Transport of Insulin Granules Is a Subordinated Random Walk, Proc. Natl. Acad. Sci. U.S.A. 110, 4911 (2013).

[11] M. J. Saxton and K. Jacobson, Single-Particle Tracking: Applications to Membrane Dynamics, Annu. Rev. Biophys. Biomol. Struct. 26, 373 (1997).

[12] A. V. Weigel, B. Simon, M. M. Tamkun, and D. Krapf, Ergodic and Nonergodic Processes Coexist in the Plasma Membrane as Observed by Single-Molecule Tracking, Proc. Natl. Acad. Sci. U.S.A. 108, 6438 (2011).

[13] F. Höfling and T. Franosch, Anomalous Transport in the Crowded World of Biological Cells, Rep. Prog. Phys. 76, 046602 (2013).

[14] J.-P. Bouchaud and A. Georges, Anomalous Diffusion in Disordered Media: Statistical Mechanisms, Models and Physical Applications, Phys. Rep. 195, 127 (1990).

[15] S. Havlin and D. Ben-Avraham, Diffusion in Disordered Media, Adv. Phys. 51, 187 (2002).

[16] J. Klafter and I. M. Sokolov, First Steps in Random Walks (Oxford University Press, Oxford, England, 2011). 
[17] M. J. Saxton, Anomalous Diffusion Due to Obstacles: A Monte Carlo Study, Biophys. J. 66, 394 (1994).

[18] J. Klafter, A. Blumen, and M. F. Shlesinger, Stochastic Pathway to Anomalous Diffusion, Phys. Rev. A 35, 3081 (1987).

[19] B. B. Mandelbrot and J. W. Van Ness, Fractional Brownian Motions, Fractional Noises and Applications, SIAM Rev. 10, 422 (1968).

[20] A. G. Cherstvy, A. V. Chechkin, and R. Metzler, Anomalous Diffusion and Ergodicity Breaking in Heterogeneous Diffusion Processes, New J. Phys. 15, 083039 (2013).

[21] P. Massignan, C. Manzo, J. A. Torreno-Pina, M. F. GarciaParajo, M. Lewenstein, and G. J. Lapeyre, Nonergodic Subdiffusion from Brownian Motion in an Inhomogeneous Medium, Phys. Rev. Lett. 112, 150603 (2014).

[22] M. V. Chubynsky and G. W. Slater, Diffusing Diffusivity: A Model for Anomalous, yet Brownian, Diffusion, Phys. Rev. Lett. 113, 098302 (2014).

[23] M. J. Saxton, Anomalous Diffusion Due to Binding: A Monte Carlo Study, Biophys. J. 70, 1250 (1996).

[24] D. Ernst, M. Hellmann, J. Köhler, and M. Weiss, Fractional Brownian Motion in Crowded Fluids, Soft Matter 8, 4886 (2012).

[25] A. V. Weigel, S. Ragi, M. L. Reid, E. K. P. Chong, M. M. Tamkun, and D. Krapf, Obstructed Diffusion Propagator Analysis for Single-Particle Tracking, Phys. Rev. E 85, 041924 (2012).

[26] A. P. Young, Spin Glasses and Random Fields (World Scientific, Singapore, 1997).

[27] E. Barkai, Aging in Subdiffusion Generated by a Deterministic Dynamical System, Phys. Rev. Lett. 90, 104101 (2003).

[28] E. Barkai, Y. Garini, and R. Metzler, Strange Kinetics of Single Molecules in Living Cells, Phys. Today 65, No. 8, 29 (2012).

[29] R. Metzler, J.-H. Jeon, A. G. Cherstvy, and E. Barkai, Anomalous Diffusion Models and Their Properties: Nonstationarity, Non-ergodicity, and Ageing at the Centenary of Single Particle Tracking, Phys. Chem. Chem. Phys. 16, 24128 (2014).

[30] A. G. Cherstvy, A. V. Chechkin, and R. Metzler, Particle Invasion, Survival, and Non-ergodicity in 2D Diffusion Processes with Space-Dependent Diffusivity, Soft Matter 10, 1591 (2014).

[31] J. Jeon, E. Barkai, and R. Metzler, Noisy Continuous Time Random Walks, J. Chem. Phys. 139, 121916 (2013).

[32] T. B. Geijtenbeek, D. S. Kwon, R. Torensma, S. J. van Vliet, G. C. van Duijnhoven, J. Middel, I. L. Cornelissen, H. S. Nottet, V. N. KewalRamani, D. R. Littman, C. G. Figdor, and Y. van Kooyk, DC-SIGN, a Dendritic Cell-Specific HIV-1-Binding Protein that Enhances Trans-infection of $T$ Cells, Cell 100, 587 (2000).

[33] Y. van Kooyk and T. B. H. Geijtenbeek, DC-SIGN: Escape Mechanism for Pathogens, Nat. Rev. Immunol. 3, 697 (2003).

[34] A. Cambi, D. S. Lidke, D. J. Arndt-Jovin, C. G. Figdor, and T. M. Jovin, Ligand-Conjugated Quantum Dots Monitor Antigen Uptake and Processing by Dendritic Cells, Nano Lett. 7, 970 (2007).
[35] A. Cambi, I. Beeren, B. Joosten, J. A. Fransen, and C. G. Figdor, The C-Type Lectin DC-SIGN Internalizes Soluble Antigens and HIV-1 Virions via a Clathrin-Dependent Mechanism, European Journal of Immunology 39, 1923 (2009).

[36] A. Sergé, N. Bertaux, H. Rigneault, and D. Marguet, Dynamic Multiple-Target Tracing to Probe Spatiotemporal Cartography of Cell Membranes, Nat. Methods 5, 687 (2008).

[37] X. Michalet, Mean Square Displacement Analysis of SingleParticle Trajectories with Localization Error: Brownian Motion in an Isotropic Medium, Phys. Rev. E 82, 041914 (2010).

[38] G. Bel and E. Barkai, Weak Ergodicity Breaking in the Continuous-Time Random Walk, Phys. Rev. Lett. 94, 240602 (2005).

[39] A. Lubelski, I. M. Sokolov, and J. Klafter, Nonergodicity Mimics Inhomogeneity in Single Particle Tracking, Phys. Rev. Lett. 100, 250602 (2008).

[40] Y. He, S. Burov, R. Metzler, and E. Barkai, Random Time-Scale Invariant Diffusion and Transport Coefficients, Phys. Rev. Lett. 101, 058101 (2008).

[41] D. Montiel, H. Cang, and H. Yang, Quantitative Characterization of Changes in Dynamical Behavior for SingleParticle Tracking Studies, J. Phys. Chem. B 110, 19763 (2006).

[42] R. Simson, E. D. Sheets, and K. Jacobson, Detection of Temporary Lateral Confinement of Membrane Proteins Using Single-Particle Tracking Analysis, Biophys. J. 69, 989 (1995).

[43] J. A. Torreno-Pina, B. M. Castro, C. Manzo, S. I. Buschow, A. Cambi, and M. F. Garcia-Parajo, Enhanced ReceptorClathrin Interactions Induced by N-Glycan-Mediated Membrane Micropatterning, Proc. Natl. Acad. Sci. U.S.A. 111, 11037 (2014).

[44] P. J. Cutler, M. D. Malik, S. Liu, J. M. Byars, D. S. Lidke, and K. A. Lidke, Multi-color Quantum Dot Tracking Using a High-Speed Hyperspectral Line-Scanning Microscope, PLoS One 8, e64320 (2013).

[45] J.-B. Masson, P. Dionne, C. Salvatico, M. Renner, C. G. Specht, A. Triller, and M. Dahan, Mapping the Energy and Diffusion Landscapes of Membrane Proteins at the Cell Surface Using High-Density Single-Molecule Imaging and Bayesian Inference: Application to the Multiscale Dynamics of Glycine Receptors in the Neuronal Membrane, Biophys. J. 106, 74 (2014).

[46] H. Feinberg, D. A. Mitchell, K. Drickamer, and W. I. Weis, Structural Basis for Selective Recognition of Oligosaccharides by DC-SIGN and DC-SIGNR, Science 294, 2163 (2001).

[47] D. A. Mitchell, A. J. Fadden, and K. Drickamer, A Novel Mechanism of Carbohydrate Recognition by the C-Type Lectins DC-SIGN and DC-SIGNR Subunit Organization and Binding to Multivalent Ligands, J. Biol. Chem. 276, 28939 (2001).

[48] A. L. Smith, L. Ganesh, K. Leung, J. Jongstra-Bilen, J. Jongstra, and G. J. Nabel, Leukocyte-Specific Protein 1 Interacts with DC-SIGN and Mediates Transport of HIV to the Proteasome in Dendritic Cells, J. Exp. Med. 204, 421 (2007). 
[49] C. Manzo, J. A. Torreno-Pina, B. Joosten, I. ReinierenBeeren, E. J. Gualda, P. Loza-Alvarez, C. G. Figdor, M. F. Garcia-Parajo, and A. Cambi, The Neck Region of the C-Type Lectin DC-SIGN Regulates Its Surface Spatiotemporal Organization and Virus-Binding Capacity on Antigen-Presenting Cells, J. Biol. Chem. 287, 38946 (2012).

[50] S. Manley, J. M. Gillette, G. H. Patterson, H. Shroff, H. F. Hess, E. Betzig, and J. Lippincott-Schwartz, High-Density Mapping of Single-Molecule Trajectories with Photoactivated Localization Microscopy, Nat. Methods 5, 155 (2008).

[51] R. A. L. Vallée, N. Tomczak, L. Kuipers, G. J. Vancso, and N. F. van Hulst, Single Molecule Lifetime Fluctuations Reveal Segmental Dynamics in Polymers, Phys. Rev. Lett. 91, 038301 (2003).

[52] G. Volpe, G. Volpe, and S. Gigan, Brownian Motion in a Speckle Light Field: Tunable Anomalous Diffusion and Selective Optical Manipulation, Sci. Rep. 4, 3936 (2014).

[53] E. Lucioni, B. Deissler, L. Tanzi, G. Roati, M. Zaccanti, M. Modugno, M. Larcher, F. Dalfovo, M. Inguscio, and G. Modugno, Observation of Subdiffusion in a Disordered Interacting System, Phys. Rev. Lett. 106, 230403 (2011).

[54] S. Krinner, D. Stadler, J. Meineke, J.-P. Brantut, and T. Esslinger, Direct Observation of Fragmentation in a Disordered, Strongly Interacting Fermi Gas, arXiv:1311.5174.

[55] B. Berkowitz, A. Cortis, M. Dentz, and H. Scher, Modeling Non-Fickian Transport in Geological Formations as a Continuous Time Random Walk, Rev. Geophys. 44, RG2003 (2006).

[56] J. Bascompte and R. Solé, Modeling Spatiotemporal Dynamics in Ecology, Environmental Intelligence Unit (Springer, New York, 1998). 